\title{
An interview with David Henry: supportive oncology, anemia and cancer
}

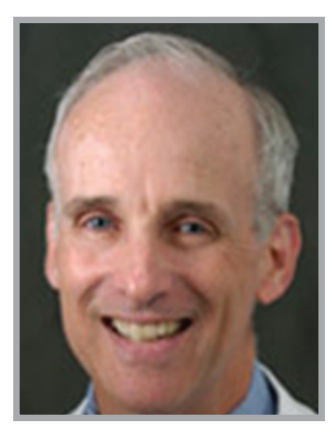

David H Henry*, MD, is a practicing hematologist/medical oncologist and Clinical Professor of Medicine at the Pennsylvania Hospital in Philadelphia (PA, USA), where he holds the title of Vice Chairman of the Department of Medicine. He is Editor in Chief of the Journal of Community and Supportive Oncology. For the past 25 years, he has had a special interest in supportive oncology and participated in clinical trials using growth factors to treat cancer-related anemia with or without intravenous iron, neutropenia, thrombocytopenia and bone metabolism due to bone metastases. He is also the Director of the HIV Malignancy Program and Director of the Austrian Medical Student Program at Pennsylvania Hospital.

Q Do you mind telling our readers about your career to date?

I went to medical school and did my hematology and oncology fellowship training at the University of Pennsylvania (PA, USA). I stayed there for most of my career (around 25 years) and I have been in two different hospitals, both in the University of Pennsylvania system. I am a general hematologist/medical oncologist, so I see many benign and malignant hematologic conditions and I am also interested in supportive care. I am involved in a number of different roles including teaching in our training hospital, in administration as Vice Chairman of the Department of Medicine and also in research within supportive care. In the USA, approximately $81 \%$ of oncology cancer care is delivered in the community, which we define as being not in the university hospital. I am technically a community oncologist because I am at a teaching affiliate of the University of Pennsylvania, not the main campus. We see so much oncology care through the community setting that we think that all community oncologists should participate in clinical research as much as possible.

\section{Q What was that initially sparked your interest in cancer \& hematologic malignancies in particular?}

I think about this as I talk to young trainees who are deciding what to go into. I was one hairs breath away from going into nephrology, but I decided to go into hematology - the difference being that sometimes patients do well and sometimes they do not, whether win or lose you always make a difference in those patients. I always liked that aspect of oncology care. The second thing was that the field was about to catch fire with regard to all the mechanisms and discoveries. Sure enough it has. The field of hematology/oncology in the US may have the most new US FDA approved drugs per year.

Q Can you tell us about your work in supportive oncology \& why this area is so important?

Supportive oncology is defined as anything that is not treating the cancer, but supporting the patient - that is, anything from treating nausea and vomiting to dehydration, diarrhea, white blood counts and red cell counts. It seemed to

*Department of Medicine, Hematology/Medical Oncology, Pennsylvania Hospital, Philadelphia, PA 19107, USA; davidhenrymd@gmail.com

Future $\because$ Medicine ${ }_{\text {part of }}$

\section{KEYWORDS \\ - anemia • iron • supportive care}


me as I got into my career and learned how to treat the cancer that there was so much else going on that was causing patients to be unhappy or to suffer. If you think about the last time you had nausea, think how awful it was for minutes, let alone days or weeks. The side effects of our great treatments can present real problems - that has interested me ever since.

Q At the Controlling Cancer Summit, London, UK, 12-14 May 2015, you discussed the use of erythropoietic stimulating agents in treating cancer/chemotherapy-induced anemia. How do you characterize chemotherapy-induced anemia in hematologic malignancies \& what problems can it present?

Women's hemoglobin should be $12 \mathrm{~g} / \mathrm{dl}$ and higher, men's should be higher due to testosterone (13 g/dl and higher). Anyone below $12 \mathrm{~g} / \mathrm{dl}$ is anemic whether you are male or female. We know from many studies in nephrology, oncology and also HIV that when hemoglobin goes down, your quality of life goes down. Parameters like fatigue and the ability to do your daily chores and think clearly are impaired as hemoglobin gets lower. The converse has been shown: as you go higher with therapy, many of those deficiencies are restored. We define chemotherapy-induced anemia as someone with cancer undergoing chemotherapy whose hemoglobin levels are below $12 \mathrm{~g} / \mathrm{dl}$.

Q What are the current strategies to manage anemia in hematologic cancer patients?

We try and ask ourselves and colleagues 'why is this patient anemic?' We cannot simply think that it is the cancer or chemotherapy, as they may be deficient in iron or vitamin B12, or there may be something wrong with the bone marrow - some patients have myelodysplastic syndromes where the bone marrow is decaying, thanks to age. Chronic renal failure can also cause anemia. We must rule out those causes and be certain that the cancer and/or chemotherapy is causing the anemia. The strategies to treat it - if we found out that it is cancer/chemotherapy-induced anemia then we really only have two strategies: if a patient is really symptomatic then there is transfusion. That is fast and we see the hemoglobin levels rise straight away. If there is time, then we can also use erythropoietic-stimulating agents (ESAs) to increase hemoglobin.
Q Would you say anemia in these patients is still undertreated?

Absolutely. In my career, I have seen it come full circle. Many years ago, we would see many patients become anemic and as doctors we would say: "Oh well it is the cancer and the chemotherapy, and the anemia goes along with the disease." Then we realized that it should not be the case. You can increase hemoglobin with transfusion or with ESAs, making a difference in improving quality of life and supportive oncology care. Then many, many worries and concerns arose about ESA safety. A few studies showed that there might be a safety issue and many areas around the world, in particular, the USA, reacted to that and reined in ESAs. While there was widespread use in the 1990s, as we entered the 2000s and especially this current decade, the use of ESAs has dropped off a lot because of the fear of a potential side effect on the patient. I think, however, that this is largely not founded.

\section{Q When should ESA treatment be given \\ \& who can it be given to?}

We want to be sure that the patient is anemic; be sure that it is not an iron, vitamin B12, folic acid, creatinine or bone marrow issue. Rule out those causes and then if a patient's hemoglobin is falling to below $11 \mathrm{~g} / \mathrm{dl}$, then you should consider starting an ESA. In the states, we have to wait until it is below $10 \mathrm{~g} / \mathrm{dl}$, but I would say at least under $11 \mathrm{~g} / \mathrm{dl}$, which is when symptoms will begin to present. This does depend on countries and insurance providers.

\section{Q What information have recent ESA studies} told us about safety \& tolerability?

This is the major question. Are they effective and safe/tolerable? There are now over 60 randomized placebo-controlled trials on using ESAs in cancer/chemotherapy - and sometimes radiotherapy-induced anemia. There were eight studies, with one of the first occurring in the UK in the 1990s, which showed that there was a safety signal of earlier death if we used higher and higher doses of ESAs to target higher and higher hemoglobin. Out of those eight, only four were in chemotherapyinduced anemia, so we now have only four where there was a safety signal. In the large bulk of trials, however, there was not. What was wrong? We first thought it might be the higher dose of ESA and/or the higher level of hemoglobin, but it is now clear that is the 
following: in the patient in whom you start an ESA for cancer/chemotherapy-induced anemia and it is not working, where hemoglobin is not rising and may be falling, then you may increase the ESA dose and may even get transfusion. Those are the patients in which the safety signal of shorter survival and possible tumor progression occur. Conversely, used on label around the world, where you start an ESA and get a response at a starting dose and one dose higher and patients are not transfused, then it is safe. If you start an ESA and it is not working despite higher ESA dose + transfusion, that is when we recommend stopping the ESA. Of course all ESAs can increase the chance for venous thromboembolism in a hazard ratio of $1: 5$.

\section{Q What is next for your research?}

We wanted to know 'does everyone respond to ESA?' and we have found that about twothirds respond and a third do not. We have been looking at why that third do not respond and whether we can get better responses like the twothirds who do. We found, as we presented at the Controlling Cancer Summit, that giving intravenous iron leads to better responses. There are more than ten randomized controlled trials of ESAs that have given intravenous iron that show the responses are better, even in the patients who already have iron. We think that what is happening is that, even though you already have iron, it may be locked up in the bone marrow because of inflammatory cytokines and cannot get to the part of the bone marrow where the red blood cells are made. Giving intravenous iron may supply that missing source and result in better responses to ESAs. Our current areas of research are in intravenous iron in anemia; and then of course there may be other ways to treat this anemia, perhaps intravenous iron only, which is possible and interesting. There is a big study occurring now, investigating whether we need ESA. Then finally, novel agents such as ACE011 are being investigated. ACE011 works via a novel mechanism on the bone marrow to increase hemoglobin and decrease transfusion in cancer patients.

Q Finally, where would you like to see the role of supportive oncology in hematologic cancer patients progress to?

That is a great question - what is our wish list in this area? I think fortunately supportive oncology is here to stay, although it was not in the beginning. Before, we did not quite see the forest through the trees and we did not see that the patient might have been miserable despite living longer. Yet, we have still not addressed many of the side effects of oncology treatments and my wish list is to address these with targeted, stronger, simpler, oral (if possible) therapies to fully address supportive oncology - just like the advancements we are seeing with immune checkpoint inhibitors and oncology therapeutics in general. I am hoping supportive oncology can catch up.

\section{Disclaimer}

The opinions expressed in this interview are those of the interviewees and do not necessarily reflect the views of Future Medicine Ltd.

Financial \& competing interests disclosure

DH Henry has consulted for Amgen and AMAG. DH

Henry has no other relevant affiliations or financial involvement with any organization or entity with a financial interest in or financial conflict with the subject matter or materials discussed in the manuscript apart from those disclosed

No writing assistance was utilized in the production of this manuscript. 